\title{
The evolving clinical picture of seronegative spondyloarthropathy based on the example of ankylosing spondylitis in a patient with a primary diagnosis of the SAPHO syndrome - case presentation
}

\author{
Corresponding author: \\ Zbigniew Guzera \\ Department of Rheumatology, \\ Saint Luke Hospital \\ Gimnazjalna 41B St. \\ 26-200 Końskie, Poland \\ tel.: + 484139002257 , \\ fax.: + 48412731958 , \\ email: zetguzera@poczta.fm
}

Medical Research Journal 2018; Volume 3, Number 3, 221-226 10.5603/MRJ.a2018.0034 Copyright (C) 2018 Via Medica ISSN 2451-2591

\begin{abstract}
Seronegative spondyloarthropathies are a group of diseases characterized by several clinical features such as inflammatory back pain, sacroiliac joint inflammation, and the presence of the HLA B27 antigen, which occurs more frequently than the general population. Non-specific bowel inflammation, skin lesions, particularly psoriasis or uveitis, are also typical in patients or their family members [1]. In many situations, clinical progression of spondyloarthropathies can be assessed by imaging of the sacroiliac joints [2, 3]. SAPHO syndrome (Synovitis, Acne, Pustulosis, Hyperostosis, Osteitis), is a rare disease, classified as seronegative spondyloarthropathy due to many typical clinical features for this group of diseases. A large variety of symptoms and atypical clinical picture of SAPHO syndrome causes significant diagnostic difficulties $[3,4]$. Presented here is a case report of a 41-year-old male patient with a twenty-year history of the disease. Initially, his disease took the form of chronic, recurrent multifocal osteomyelitis (CRMO), which finally turned into a spondyloarthropathy that met the classification criteria for ankylosing spondylitis. Key words: seronegative spondyloarthropathy, ankylosing spondylitis, SAPHO syndrome, osteomyelitis, CRMO, spondylodiscitis
\end{abstract}

Med Res J 2018; 3 (4): 221-226

\section{INTRODUCTION}

SAPHO syndrome is a rare clinical syndrome with co-occurrence of synovitis, skin lesions such as acne, palmoplantar pustulosis (PPP), excessive bone formation and osteomyelitis. According to current knowledge, it is not possible to clearly determine the pathogenetic mechanisms of the disease. Environmental factors, e.g. infections (most often Propionibacterium acnes), or a genetic predisposition, have been considered $[2,4]$. The inclusion of SAPHO syndrome into spondyloarthropathies is supported by the presence of sacroiliitis, vertebral osteomyelitis, discitis, psoriasis and inflammatory bowel diseases that occur more frequently than in the general population, similarly to the presence of HLA B27 antigen, which occurs in about $30 \%$ of cases [2-4]. According to some authors, there are two subtypes of SAPHO syndrome, one of which is a form of spondyloarthropathy coexisting with hyperostosis and skin lesions, mostly with morphology of palmo-plantar pustulosis or pustular psoriasis [2]. This form is dominated by symptoms of arthritis in the anterior chest wall and inflammation of the sternocostal joints [2, 5]. Inflammatory changes also often occur in the sacroiliac joints, spinal joints and/or intervertebral discs as well as in of hands and feet joints, often asymmetrically $[1,2,5]$. The other type presents with chronic, recurrent, multifocal inflammation of the bone and bone marrow (CRMO), also defined as CNO (chronic, non-bacterial osteomyelitis) $[2,6]$. This syndrome, until recently, has been mainly observed in children and young adults, and is characterized by recurring inflammation of the 
Table 1. Diagnostic criteria of SAPHO syndrome according to Kahn $[7,10]$

Chronic and recurring inflammations of bone and bone marrow (CRMO/CNO)

- Usually aseptic (seldom propionibacterium acne in PCR)

- with the involvement of the spine and peripheral bones

- with or without skin lesions

Aseptic, acute or chronic arthritis coexisting with:

- pustular psoriasis of hands and feet (PPP)

- hidradenitis

- acne (conglobata, ulcerans)

Each inflammation of bones and marrow coexisting with :

- pustular psoriasis of hands and feet (PPP)

- hidradenitis

- acne (conglobata, ulcerans)

bones and/or bone marrow, especially in the epiphyses of long bones (femur and tibia), intervertebral discs, collarbones, mandible and the pelvis [7-9].

The diagnostic criteria of SAPHO syndrome were presented in 1994 by Kahn (Tab.1). Meeting one of the proposed criteria is necessary in order to diagnose the syndrome $[7,10]$.

SAPHO syndrome diagnosis is difficult due to the frequently atypical clinical picture, the occurrence of different symptoms at various stages of the disease, and the lack of a specific biomarker. However, specific changes are often found in imaging studies [4]. The most frequently observed laboratory abnormalities are elevated ESR and CRP or increased activity of alkaline phosphatase [2].

In imaging studies, subchondral bone sclerosis may be observed, as well as erosions in the anterior chest wall joints, sometimes with periosteal inflammation. Pericostal ossification and hyperostosis are frequent phenomena, particularly in the collarbone area, as well as osteosclerosis of bone bodies, osteophytosis and syndesmophytes in the thoracic and lumbosacral spine $[2,5]$. Inflammation of the sacroiliac joints occurs in about $35 \%$ of the cases. [3, 7] Inflammation of intervertebral discs is found in about $25-35 \%$ of patients and is also part of the spine changes related to the disease.

Imaging is very helpful for SAPHO syndrome diagnosis and differentiation between the observed structural changes. Classical radiography, computed tomography, scintigraphy and magnetic resonance (especially in early stages of the disease) are used. Histopathological tests are essential to determine the nature of bone tissue changes $[5,8,10]$.

Differential diagnostics is based, mainly, on the exclusion of an underlying infection of the bones and/or bone marrow, proliferative processes, or histiocytosis $[2,5]$. In the diagnosis of SAPHO syndrome, it has become crucial to consider diseases such as ankylosing spondylitis, psoriatic arthritis or arthritis accompanying inflammatory bowel diseases. All of these conditions are characterized by the similarities of symptoms, as well as a potential evolution of the clinical picture during the course of the disease [2, 3].

This paper discusses the case of a 41-year-old male patient with a long-term history of osteomyelitis symptoms and inflammation of the intervertebral discs, in whom symptoms of spondyloarthropathy developed over the last twenty years of the disease. The patient on admission to the Rheumatology Ward, fulfilled the American College of Rheumatology (ACR) modified New York criteria of ankylosing spondylitis, established in1988.

\section{Case description}

We presented the case of a 41 -year-old male patient with a history of recurrent episodes of inflammation of the body and left ramus of the mandible, occurring since the age of 19. Inflammation of intervertebral discs and progressive, multilevel ossification of the thoracic and lumbar spine segments was observed in the subsequent years. He was admitted to the Rheumatology Ward with significantly increased pain in the spine and hip joints, which remained constant despite the time of day, physical activity, and experienced long-lasting morning stiffness. He had no history of comorbidities, and no significant family history for any diseases, important for further diagnosis. His multiannual history required retrospective analysis of medical records provided, concerning the years between 1992 and 2013. The occurrence of inflammation of the mandible, which appeared between 1992 and 1995, and affected the body and the left mandible ramus, was described there. During several hospital admissions to the dental surgery clinic, laboratory tests showed only a slight elevation in inflammatory markers. X-ray of the mandible was described as: "several osteolytic foci and necroses within the body and the left mandibular ramus". Histopathological assessment in 1995 (during the third year of the disease), corresponded to chronic inflammation of the bone with possible complications related to fibrous dysplasia; intertrabecular bone remodelling, multiplication of fibrous tissue and vast inflammatory infiltrations were also described. Multiple antibiotic therapies (including lincomycin, vancomycin, tobramycin, pefloxacin, sulfamethoxazole with trimethoprim, oxacillin, amoxicillin with clavulanic acid), and non-steroidal anti-inflammatory drugs (NSAIDs) were administered to this patient several times between 1992 and 1995. In the subsequent years, no recurrences of the inflammatory changes of the jaw bone were observed. He had his teeth extracted on multiple occasions due to advanced decay. 
Back pain, without typical symptoms of inflammation, as well as progressive limitation of thoracic spine mobility, became dominant in the clinical picture since 2006, which forced the patient to take NSAID that exceeded the maximum doses. This led to recurring erosive gastritis with mild anaemia, in subsequent years. MRI tests showed hyperintense areas of the end plates of TH12-L5 bodies in the T-1 and T2-weighted images that, according to the description, "correspond to Scheuermann's disease or bone fragility due to metabolic reasons".

Owing to the suspicion of a proliferative disease, the patient was extensively examined, including undergoing a bone marrow histopathological assessment in the oncology dept., where the neoplastic disease was excluded.

Due to the increasing pain and further reduction in the spine mobility after the subsequent 7 months, the patient was admitted in the rheumatology ward. There, the progression of thoracic spine changes in the MRI with uneven contours of the end-plates and losses at the levels of Th10/Th11/Th12 were found. Compression of Th12 body with marginal osteosclerosis and similar, multilevel changes in the lumbar part were also described. In the radiologist's opinion, this picture supported an inflammatory background, most probably haematogenous. During this time, no inflammatory changes in the sacroiliac joints were found. Laboratory tests showed ESR $72 \mathrm{~mm}$, CRP 157mg/l, Hb $10.4 \mathrm{G} / \mathrm{dL}$, and ALP $409 \mathrm{U} / \mathrm{L}$, characteristic for an active inflammatory process. The high level of alkaline phosphate was assessed as not linked to liver pathology. HLA B27 antigen detection and the TST (tuberculin skin test/Mantoux test) were negative. Following neurosurgical consultation, the patient was transferred to the neurosurgery ward with a diagnosis of infectious spondylodiscitis. There, antibiotic therapy, subcutaneous administration of calcitonin, and a plaster cast corset were applied. In subsequent years' constant spinal pain in the thoracic and lumbar areas continued. As well as kyphoscoliosis, deterioration of the strength of his lower extremities occurred.

In 2010, subsequent MRI of the thoracic spine described: "...there is most probable an inflammatory mass surrounding the vertebral bodies from the front and lateral side with a $12 \mathrm{~mm}$ thick muff along the thoracic spine, penetrating and surrounding the costovertebral joints and transverse processes, there is an inflammatory infiltration in the epidural space, the spinal cord is compressed" showing the progression of the inflammatory changes and risk of occurrence of neurological symptoms resulting from the compression of the spinal cord. This, alongside consistently elevated ESR, prompted subsequent antibiotic therapy.
Spastic paresis of the muscles occurred in this patient in 2013. A multilevel laminectomy procedure was performed in the neuro-orthopaedics dept. Intraoperative biopsy and inflammatory tissue culture were performed during the procedure and revealed no bacterial growth. The subsequent MRI showed vast ossification of the majority of motion segments in the cervical, thoracic and lumbar spine. This clinical picture was deemed as a consequence of advanced ankylosing spondylitis or vast, chronic, infectious inflammatory changes. During hospitalization, SPECT (single-photon emission computed tomography) examination was performed, and accumulation of a marker in the thoracic spine, heel bones, pelvis and the sacroiliac joints were observed.

There was no clinical improvement in subsequent months, despite daily treatment with NSAID (400 mg of ketoprofen), and $400 \mathrm{mg}$ of tramadol. Increasing pain and decreased mobility of the lumbosacral spine and pelvis resulted in the patient becoming wheelchair bound. He returned to the rheumatology ward in April 2014.

Physical examination showed intense pain and multidimensional limitation of mobility in the thoracic and lumbosacral spine, as well as in the hip joints. Tests assessing the sacroiliac joints were uninterpretable due to hip joints involvement. There were no inflammatory symptoms in other peripheral joints. During the physical examination, no cutaneous or nail lesions, no digestive tract involvement symptoms, as well as any other significant findings, were observed. Numerous abnormalities were found in laboratory tests: ESR $58 \mathrm{~mm} / \mathrm{h}, \mathrm{CRP}$ $81.8 \mathrm{mg} / \mathrm{L}$, ALP $772 \mathrm{U} / \mathrm{L}, \mathrm{Hb} 12.8 \mathrm{~g} / \mathrm{dL}$, hypergammaglobulinemia, with no presence of monoclonal protein, low 250HD concentration (25-hydroxy-vitamin D), decreased urinary calcium excretion, increased type 1 C-terminal telopeptide and osteocalcin, increased testosterone concentration, normal concentration of the parathyroid hormone. Rheumatoid factor and anti-citrullinated peptide antibodies, as well as anti-nuclear antibodies, were absent. The Quantiferon Gold test and Brucella antibodies were negative.

In the X-ray of the spine (Fig. 1), multilevel syndesmophytes (picture of a "bamboo spine") in the thoracic and lumbosacral sections of the spine were demonstrated, in addition to lesions related to the laminectomy. Pelvis X-ray (Pic.2) showed remodelling of the bone structure, uneven surfaces of the femoral heads, protrusion, and significant bilateral hip joint space narrowing. In the sacroiliac joints, narrowed, blurred and uneven joint spaces with subchondral bone sclerosis were found.

MRI of the pelvis revealed narrowing and blurred sacroiliac joint spaces (mainly in the lower part of the joint), with periarticular bone marrow oedema in the sacrum and ilium, which was enhanced with contrast 


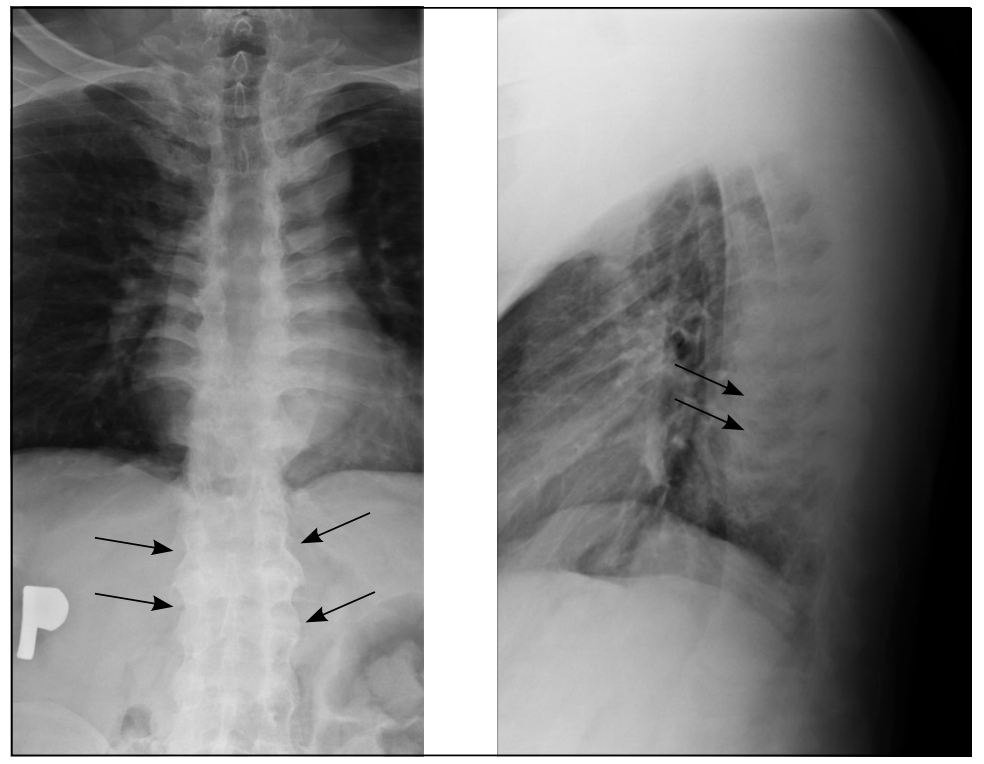

Figure 1. Changes in the spine of the „bamboo spine” type (arrows)

administration. Additionally, bilateral deepening of the acetabulum, with the femoral head bulging into the small pelvis were described. Bilateral deformation of the head and neck of the femur and bone remodelling were also found. Also described were reduced signal from the pelvic cartilage with bone ankylosis, effusion in the periarticular bursae and synovial overgrowth. The clinical picture corresponded to the ankylosing spondylitis accompanied by hip joint inflammation.

The full doses of NSAID, opioid analgesics, including buprenorphine, myorelaxants were implemented. Vitamin D3 supplementation was also applied. BASDAI (Bath Ankylosing Spondylitis Activity Score) index was scored as 5.8. The spine pain VAS (Visual Analogue Scale) was scored as $76 \mathrm{~mm}$.

After supplementing vitamin $\mathrm{D}$ deficiency, the patient was administered intravenous infusion of $90 \mathrm{mg}$ Pamidronate. However, the patient refused continuation of this treatment due to lower extremity pain and malaise.

During treatment, clinically significant improvement was not observed. Follow-up BASDAI after monthly therapy according to the above schedule was scored 8.9, and pain of the spine VAS was checked as $90 \mathrm{~mm}$.

According to the observed activity of the disease and risk of complete loss of hip structures, treatment with an anti-TNF-alpha drug was started. The patient was administered with $50 \mathrm{mg}$ of golimumab, and a significant reduction in the symptoms of the inflammatory disease was achieved within 3-months. During the regular assessments of the therapy course, there were a reduction in ESR values, CRP concentration and improvement in BASDAI and VAS values of spinal pain (Tab.2).

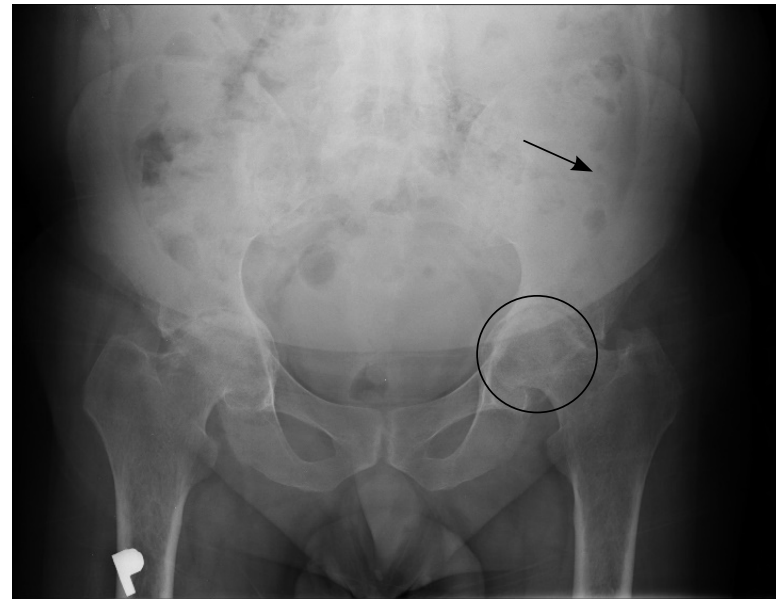

Figure 2. Inflammatory changes in the sacroiliac joints (arrows). Inflammatory changes in the pelvis (ring)

During anti-TNF-alpha therapy, no side effects were observed. Treatment was stopped due to low disease activity in the $18^{\text {th }}$ month. However, after about 8 weeks the patient suffered from a recurrence disease activity symptoms (shown by the highlighted line in Table 2.). An improvement in laboratory parameters was achieved and low disease activity was observed after therapy with golimumab restarting on the 23 rd week of treatment.

\section{Discussion}

Diagnosis of seronegative spondyloarthropathy, according to the criteria of ASAS (Assessment of Spondy- 
Table 2. List of assessments of the disease activity in subsequent months of anti-TNF- alpha therapy. Highlighted the line of the table presents the assessment after the treatment with golimumab discontinuation

\begin{tabular}{lcccc}
\hline Month of therapy & ESR & CRP & VAS of the spine pain & BASDAI \\
\hline 3 & $4 \mathrm{~mm} / \mathrm{h}$ & $9.4 \mathrm{mg} / \mathrm{L}$ & $60 \mathrm{~mm}$ & 3.40 \\
6 & $5 \mathrm{~mm} / \mathrm{h}$ & $4.2 \mathrm{mg} / \mathrm{L}$ & $30 \mathrm{~mm}$ & 3.10 \\
18 & $2 \mathrm{~mm} / \mathrm{h}$ & $6.5 \mathrm{mg} / \mathrm{L}$ & $15 \mathrm{~mm}$ & 2.20 \\
20 & $59 \mathrm{~mm} / \mathrm{h}$ & $121 \mathrm{mg} / \mathrm{L}$ & $80 \mathrm{~mm}$ & 6.60 \\
23 & $8 \mathrm{~mm} / \mathrm{h}$ & $9.5 \mathrm{~g} / \mathrm{dL}$ & $10 \mathrm{~mm}$ & 1.40 \\
\hline
\end{tabular}

loArthritis International Society) from 2010 is possible on the basis of the typical clinical symptoms, co-occurring with (often discrete) imaging changes, or the presence of HLA B27 antigen [2, 3]. Many symptoms typically related to spondyloarthropathies, such as the spine joints or the sacroiliac joints inflammation, coexisting psoriasis or the inflammatory bowel disease, justify the classification of SAPHO syndrome into this group [2]. Similarly, like many other spondyloarthropathies, SAPHO syndrome can also evolve in its course. Changes in the involved joints, develop over the years. In some cases, the patient initially diagnosed as SAPHO syndrome, during the course of the disease, fulfil the modified New York diagnostic criteria for ankylosing spondylitis, or classification criteria of CASPAR (Classification Criteria for Psoriatic Arthritis) [3, 11].

SAPHO syndrome is a rare disease, and often its atypical course, "stretching over the time" of particular symptoms, as well as a lack of specific biomarkers of the disease, makes the correct diagnosis difficult to establish. According to related publications, there is often a long diagnostic delay for cases of SAPHO syndrome [4, 7]. Diagnostic difficulties may particularly refer to cases which run as chronic, recurring (in many cases recurrences can be easily overlooked), multifocal osteomyelitis (CRMO). Vertebral bodies and the intervertebral discs or, as in this case, the mandible, are frequent locations of the inflammatory changes. An acute course of the disease often presents with inflammation of intervertebral discs, which can lead to a significant deterioration in the patient's mobility, especially, if there are complications, such as spinal cord compression [6]. Laboratory abnormalities accompanying the disease, and the observed imaging changes may suggest a developing proliferative process or infectious etiology. As a result, it often causes complex, time- and cost-consuming diagnostics being performed, or exposes patients to long-term antibiotic therapy. [12-14].

In the present state of knowledge, there are no uniform SAPHO syndrome treatment recommendations developed [15]. NSAIDs are commonly used and in many cases are effective as the disease course is often a relatively mild. In many situations, particularly with coexisting skin lesions, antibiotics, for example, azithromycin or doxycycline are used in therapy. Disease-modifying antirheumatic drugs (DMARDs), such as methotrexate or sulfasalazine are also useful in cases when the disease manifests in peripheral joints, however, information on their effectiveness varies $[14,15]$. Many bisphosphonate studies demonstrate good results of such therapy, but they are rather limited to reduce the extent of bone changes. In publications, intravenous administration of pamidronate in doses between 30 to $90 \mathrm{mg}$ is most frequently described with positive results reported [16]. Glucocorticosteroids play a relatively insignificant role, similarly to other spondyloarthropathies. Their application is limited to local or oral administration in acute presentations of the disease [17]. In recent years there have been some publications discussing the positive effects of SAPHO syndrome therapy with anti-TNF-alpha agents $[18,19]$. Most frequently, publications describing positive results with this group refer to Infliximab, administered in a typical for spondyloarthropathies dose, $5 \mathrm{mg} / \mathrm{kg}$ of body weight at 0,2 and 6 weeks $[19,20]$. Unfortunately, along with increased use of TNF-alpha inhibitors for SAPHO syndrome, there are a number of the publications revealing an increased risk of exacerbation of skin manifestations, which suggests their distinctness from osteoarticular symptoms [21].

\section{Conclusions}

Presented case is an example of the SAPHO syndrome, commencing with typical CRMO symptoms which, over time evolving into seronegative spondyloarthropathy, in the course of which the clinical symptoms and advanced imaging changes allow diagnose of disease as ankylosing spondylitis. Symptoms of the mandible and intervertebral disc inflammation required the exclusion of a proliferative process or an infectious disease. Repeated, with no positive result antibiotic therapy is characteristic in the history of this patient, similarly to other cases described. Special emphasis 
should be put on the fact of non-occurrence of psoriasis or above-mentioned skin lesions, which often coexist with SAPHO syndrome, the absence of symptoms of other diseases, such as inflammatory bowel disease or uveal diseases, in the patient as well as in his family history. The absence of HLA B27 antigen in this patient, despite various symptoms of ankylosing spondylitis, is also worth mentioning.

In this case, the decision to administer TNF-alpha inhibitor was a result of the disease evolution to the typical clinical picture of ankylosing spondylitis. Involvement of the hips, which could result in a complete destruction of these joints' structures, as well as the total ineffectiveness of the initial treatment, predisposed patient to a risk of permanent disability. Observing the clinical response to golimumab administration, and particularly the rapid recurrence of the symptoms after the therapy interruption, which is commonly observed during therapy of spondyloarthropathies with anti-TNF alfa agents, seem to confirm, that this case describes an evolution of typical SAPHO syndrome to ankylosing spondylitis.

\section{Disclosure of interest: Authors report no competing interests}

\section{Abbreviations}

ACR - American College of Rheumatology

ALP - alkaline phosphatase

ASAS - Assessment of Spondyloarthritis International

Society

BASDAI - Bath Ankylosing Spondylitis Activity Score

CASPAR - Classification Criteria for Psoriatic Arthritis

CNO - chronic, non-bacterial osteomyelitis

CRMO - chronic, recurrent multifocal osteomyelitis

CRP - C-reactive protein

ESR - erythrocyte sedimantation rate

$\mathrm{Hb}$ - hemoglobin

HLA B27 - Human Leukocyte Antigen B27

$\mathrm{MRI}$ - magnetic renosance imaging

NSAIDs - non-steroidal anti-inflammatory drugs

$\mathrm{PCR}$ - polymerase chain reaction

PPP - palmo-plantar pustulosis

SAPHO - Synovitis, Acne, Pustulosis, Hyperostosis,

Osteitis

SPECT — single-photon emission computed tomography

TNF alpha - tumor necrosis factor alpha
TST — tuberculin skin test

VAS - Visual Analogue Scale

\section{References}

1. Nguyen MT, Borchers A, Selmi C, et al. The SAPHO syndrome. Semin Arthritis Rheum. 2012; 42(3): 254-265, doi: 10.1016/j.semarthrit.2012.05.006, indexed in Pubmed: 23153960.

2. Przepiera-Będzak H, Brzosko M, Brzosko I. Zespół SAPHO. Pol Arch Med Wewn. 2004; 111(2): 265-8.

3. Van Mechelen M, Lories RJ. Microtrauma: no longer to be ignored in spondyloarthritis? Curr Opin Rheumatol. 2016; 28(2): 176-180, doi: 10.1097/BOR.0000000000000254, indexed in Pubmed: 26751839.

4. Van Doornum S, Barraclough D, McColl G, et al. SAPHO: rare or just not recognized? Semin Arthritis Rheum. 2000; 30(1): 70-77, doi: 10.1053/sarh.2000.8371, indexed in Pubmed: 10966214.

5. Schilling F. [SAPHO syndrome: clinico-rheumatologic and radiologic differentiation and classification of a patient sample of 86 cases]. Z Rheumatol. 2000; 59(1): 1-28, indexed in Pubmed: 10769419.

6. Kubaszewski $\measuredangle$, Wojdasiewicz P, Rożek M, et al. Syndromes with chronic non-bacterial osteomyelitis in the spine. Reumatologia. 2015; 53(6): 328-336, doi: 10.5114/reum.2015.57639, indexed in Pubmed: 27407266.

7. Kahn MF, Khan MA. The SAPHO syndrome. Baillieres Clin Rheumatol. 1994; 8(2): 333-362, indexed in Pubmed: 8076391.

8. Fleuridas G, Teysseres N, Ragot JP, et al. [Diffuse sclerosing osteomyelitis of the mandible and SAPHO syndrome]. Rev Stomatol Chir Maxillofac. 2002; 103(2): 96-104, indexed in Pubmed: 11997737.

9. Kahn MF, Hayem F, Hayem G, et al. Is diffuse sclerosing osteomyelitis of the mandible part of the synovitis, acne, pustulosis, hyperostosis, osteitis (SAPHO) syndrome? Analysis of seven cases. Oral Surg Oral Med Oral Pathol. 1994; 78(5): 594-598, indexed in Pubmed: 7838465.

10. Zielińska A, Rupiński R, Filipowicz-Sosnowska A. Zespół SAPHO - odmienność przebiegu - trudności diagnostyczne. Reumatologia. 2006; 44: 213-219.

11. Dumolard A. Gaudin Ph. Sapho syndrome or psoriatic arthritis? Rheumatology. 1998; 38: 463-7.

12. Olivieri I, Padula A, Palazzi C. Pharmacological management of SAPHO syndrome. Expert Opin Investig Drugs. 2006; 15(10): 1229-1233, doi: 10.1517/13543784.15.10.1229, indexed in Pubmed: 16989598

13. Rukavina I. SAPHO syndrome: a review. J Child Orthop. 2015; 9(1): 19 27, doi: 10.1007/s11832-014-0627-7, indexed in Pubmed: 25585872.

14. Govoni M, Colina M, Massara A, et al. „SAPHO syndrome and infections". Autoimmun Rev. 2009; 8(3): 256-259, doi: 10.1016/j. autrev.2008.07.030, indexed in Pubmed: 18721907.

15. Özen M, Kalyoncu U. SAPHO syndrome may be treated effectively with combined drug regimens - A case report. International Journal of Case Reports and Images. 2011; 2(1): 8, doi: 10.5348/ijcri-2011-01-14-cr-2.

16. Courtney PA, Hosking DJ, Fairbairn KJ, et al. Treatment of SAPHO with pamidronate. Rheumatology (Oxford). 2002; 41(10): 1196-1198, indexed in Pubmed: 12364646.

17. Delattre E, Guillot X, Godfrin-Valnet M, et al. SAPHO syndrome treatment with intravenous pamidronate. Retrospective study of 22 patients. Joint Bone Spine. 2014; 81(5): 456-458, doi: 10.1016/j. jbspin.2014.01.017, indexed in Pubmed: 24561020.

18. Wagner $A D$, Andresen J, Jendro MC, et al. Sustained response to tumor necrosis factor alpha-blocking agents in two patients with SAPHO syndrome. Arthritis Rheum. 2002; 46(7): 1965-1968, doi: 10.1002/art.10539, indexed in Pubmed: 12124882

19. Ben Abdelghani K, Dran DG, Gottenberg JE, et al. Tumor necrosis factor-alpha blockers in SAPHO syndrome. J Rheumatol. 2010; 37(8): 16991704, doi: 10.3899/jrheum.091086, indexed in Pubmed: 20472920

20. Hayem G, Ben M', Toussirot E, et al. SAPHO syndrome treated by TNF alpha-blocking agents: report of 45 cases. Arthritis Rheum. 2010; 62: S2269.

21. Baeten D, van Hagen PM. Use of TNF blockers and other targeted therapies in rare refractory immune-mediated inflammatory diseases: evidence-based or rational? Ann Rheum Dis. 2010; 69(12): 2067-2073, doi: 10.1136/ard.2009.126813, indexed in Pubmed: 20705637. 